\title{
L'approvazione e il rimborso delle nuove procedure di aferesi terapeutica
}

\author{
Carmelo Loschiavo
}

U.O.C. Nefrologia e Dialisi, Ospedale Civile, Legnago (VR)

\begin{abstract}
The APPROVAL AND REIMBURSEMENT OF NEW THERAPEUTIC APHERESIS PROCEDURES
Abstract. Therapeutic apheresis is approved as an essential therapy in many therapeutic procedures and in the guidelines of several scientific societies. In contrast to what happens in many Western Countries, in Italy therapeutic apheresis is not regulated by an adequate legislation for the modern needs. This gap in the national healthcare system has been filled in some regions, only partially and in different ways, by issuing LEA confined to the territory of the single region. This has created a clear inequality in patients' treatment depending on their region of residence; to overcome this issue, the Italian Society of Nephrology and the Italian Society of Hemapheresis have proposed, togheter, a standard for patients' identification and charging that allows leveling out reimbursement and feasibility of this procedure on the whole national territory.
\end{abstract}

Key words: Therapeutic apheresis, NHS, Reimbursement procedures

Conflict of interest: None.

Financial support: None.

Ricevuto: 10 Gennaio 2013; Accettato: 27 Febbraio 2013

L'aferesi terapeutica, pur essendo ormai considerata una terapia essenziale in molte patologie e, in alcune di esse, tipo l'iperlipemia familiare, addirittura una "terapia salvavita", in Italia è orfana di una chiara normativa nazionale nell'ambito delle prestazioni fornite dal SSN.

Al contrario di altri paesi industrializzati, in cui il problema è stato risolto da anni (Giappone 1986, USA 1996, Germania 1991-2003, Regno Unito e Francia 2004), nel nostro Paese, invece, la situazione è talmente caotica da pregiudicare la stessa possibilità di accedere a questa terapia in modo omogeneo sull'intero ambito nazionale per tutti i pazienti nei quali vi è l'indicazione clinica, creando, di fatto, una disuguaglianza di trattamento legata alla residenza e, quindi, disattendendo il Mandato Costituzionale che sancisce parità di trattamento per tutti i cittadini della Repubblica. Infatti, nel Nomenclatore Nazionale, vi è solo la voce plasmaferesi (PEX) con tariffazione pari a Euro 438.50; l'introduzione della LDL-aferesi si è avuta nella G.U. del 12/08/97 e la sua associazione all'elenco delle malattie croniche (Iperlipemia Familiare) con la G.U. del 25/09/99, per essere, infine, riconosciuta come prestazione salvavita erogabile, nell'ambito delle malattie rare, esente dalla partecipazione alla spesa della prestazione solo per i pazienti con codice patologia 025 , nella G.U. del 12/07/2001. Il tutto, purtroppo, lasciando intatta la tariffazione di rimborso appiattita sulla voce plasma- feresi e, quindi, non riconoscendo neanche la totale diversità clinica e operativa, oltre che di costi, dell'aferesi terapeutica rispetto alla classica PEX. Alcune Regioni, agendo singolarmente senza alcun coordinamento e in maniera fra loro difforme, hanno parzialmente colmato la lacuna inserendo la LDL-aferesi fra i propri LEA regionali (Tab. I). Se poi, dal campo della LDL-aferesi, si passa a tutte le altre metodiche di aferesi terapeutica (adsorbimento, immunoassorbimento su colonna, filtrazione a cascata), la cui efficacia è talmente comprovata da essere contemplate anche nelle Linee Guida terapeutiche di diverse Società Scientifiche e utilizzabili in un ampio scenario clinico, comprendente patologie su base immunologica, patologie vascolari su base aterosclerotica, esecuzione di trapianto di rene in pazienti immunizzati e così via, il Nomenclatore Nazionale delle prestazioni erogabili dal SSN non fa neanche menzione di tutto ciò, appiattendo il tutto alla sola voce "plasmaferesi" summenzionata, ma con l'aggravante che il divario tra costo della prestazione e suo rimborso è ancora più eclatante, tale da impedire o, almeno, scoraggiare le velleità di approntare e rendere disponibile la terapia in gran parte del nostro territorio. Anche per questo vi sono state iniziative regionali autonome, sulla falsariga della LDL-aferesi, a "macchia di leopardo" (Tab. II). Al Maggio del 2011 risale il tentativo di inserimento nel Nomenclatore Nazionale delle metodiche di aferesi terapeutica, con la 
L'approvazione e il rimborso delle nuove procedure di aferesi terapeutica

TABELLA I - LEA REGIONALI AGGIUNTIVI PER L'EROGAZIONE NEL PROPRIO TERRITORIO DELLA LDL-AFERESI

\begin{tabular}{lll}
\hline Regione & Rimborso & Note \\
\hline Valle d'Aosta & 1500 Euro & \\
Veneto & 929 Euro & Richiesta x ciclo (max n. 6 sedute) \\
Lombardia & 1500 Euro & Richiesta x singola prestazione \\
Toscana & 1500 Euro & Solo centri ospedalieri specializzati \\
Piemonte & 516 Euro & Supplemento di 607.60 Euro se con immunoassorbimento \\
Lazio & $\begin{array}{l}\text { Non inserite nel Nomenclatore Regionale; } \\
\text { rimborso a piè di lista }\end{array}$ & $\begin{array}{l}\text { Autorizzato solo Policlinico Umberto I per LdL-aferesi e aferesi } \\
\text { selettiva }\end{array}$ \\
Abruzzo & 774.69 Euro & Assimilati (PEX, LDL selettiva, filtrazione a cascata) \\
Sicilia & 1500 Euro & Solo Centri Regionali autorizzati \\
\hline
\end{tabular}

TABELLA II - LEA REGIONALI AGGIUNTIVI PER L'EROGAZIONE NEL PROPRIO TERRITORIO DELLE VARIE METODICHE DI AFERESI TERAPEUTICA

\begin{tabular}{|c|c|c|}
\hline Regione & Rimborso & Note \\
\hline Piemonte & 1150 Euro & \\
\hline Veneto & 1796 Euro & $\begin{array}{l}\text { LEA Regionale da erogarsi ai residenti in Veneto o ai pazienti extra } \\
\text { Regione, previa autorizzazione ULSS di residenza }\end{array}$ \\
\hline Toscana & 665 Euro & Per fotoaferesi extracorporea \\
\hline Lombardia & 628 Euro & Sotto la voce plasmaferesi terapeutica \\
\hline Abruzzo & 774.69 Euro & Qualsivoglia metodologia aferetica \\
\hline Lazio & $\begin{array}{l}\text { Non inserite nel Nomenclatore Regionale; } \\
\text { rimborso a piè di lista }\end{array}$ & $\begin{array}{l}\text { Autorizzato solo Policlinico Umberto I per LdL-aferesi e aferesi } \\
\text { selettiva }\end{array}$ \\
\hline Sicilia & $\begin{array}{l}\text { Filtrazione a cascata } 1500 \text { Euro } \\
\text { Colonna assorbimento } 1248 \text { Euro } \\
\text { Immunoassorbimento } 1248 \text { Euro } \\
\text { Fotoaferesi } 1537 \text { Euro }\end{array}$ & Solo Centri Regionali autorizzati \\
\hline
\end{tabular}

relativa tariffa di rimborso, al fine di uniformare il tutto e di porre fine alla palese sperequazione di trattamento derivante dalla "latitudine di residenza", mediante una proposta comune formulata congiuntamente dalla Società Italiana di Emaferesi e Manipolazione Cellulare (SIdEM) e dalla Società Italiana di Nefrologia (SIN) (1); non vi è stata, però, alcuna risposta da parte delle Autorità Ministeriali. In considerazione della capillarità dei centri di dialisi e della dimestichezza dei Nefrologi nel maneggiare le diverse e sofisticate tecniche depurative extracorporee, di cui l'aferesi terapeutica rappresenta una fra le tante e, per di più, tecnicamente tra le più semplici da eseguire, dai Nefrologi stessi, insieme ai colleghi Trasfusionisti, dovrebbe partire con più vigore la richiesta di ammodernamento del Nomenclatore del SSN, con le indicazioni precise all'utilizzo della metodica, ricavabili anche dall'ultima Consensus Conference italiana (2), al fine di consentire a tutti i pazienti di beneficarne, applicando il dovere Costituzionale della Repubblica di rimuovere gli ostacoli di ordine economico e sociale che limitano di fatto l'uguaglianza tra i cittadini.

\section{Riassunto}

L'aferesi terapeutica, riconosciuta come terapia essenziale in molte procedure terapeutiche e presente nelle Linee Guida di diverse Società Scientifiche, in Italia non ha una normativa adeguata alle esigenze moderne, al contrario di quanto avviene in molti paesi occidentali. La lacuna del Sistema Sanitario Nazionale è stata colmata parzialmente e in modo difforme in alcune Regionali, mediante l'emanazione di propri LEA limitati al territorio della singola Regione. Ciò ha creato una netta disu 
guaglianza di trattamento per i pazienti, a seconda della loro residenza, e, per superare ciò, le Società di Nefrologia e di Emaferesi hanno proposto congiuntamente uno standard di identificazione e tariffazione delle pratiche di aferesi terapeutica che livelli il rimborso e la fattibilità di tale prestazione su tutto il territorio nazionale.

Parole chiave: Aferesi terapeutica, Nomenclatore tariffario, Procedure rimborsi

Dichiarazione di conflitto di interessi: L'Autore dichiara di non avere conflitto di interessi.

Contributi economici degli autori: L'Autore dichiara di non aver ricevuto sponsorizzazioni economiche per la preparazione dell'articolo.
Indirizzo degli Autori:

Dr. Carmelo Loschiavo

U.O.C. Nefrologia e Dialisi

Ospedale Civile

Via Gianella 1

37047 Legnago (VR)

carmelo.loschiavo@aulsslegnago.it

\section{Bibliografia}

1. Proposta di revisione del Nomenclatore Nazionale relativamente alle procedure di aferesi terapeutica. G Ital Nefrol 2011; 28 (6): 672-5.

2. II Consensus Conference italiana sulla LDL-aferesi. 2009; 15 Maggio, Roma. 